\title{
A Simple Proof of Threshold Saturation for Coupled Vector Recursions
}

\author{
Arvind Yedla, Yung-Yih Jian, Phong S. Nguyen, and Henry D. Pfister \\ Department of Electrical and Computer Engineering, Texas A\&M University
}

\begin{abstract}
Convolutional low-density parity-check (LDPC) codes (or spatially-coupled codes) have now been shown to achieve capacity on binary-input memoryless symmetric channels. The principle behind this surprising result is the threshold-saturation phenomenon, which is defined by the beliefpropagation threshold of the spatially-coupled ensemble improving to a fundamental threshold defined by the uncoupled system.

Previously, the authors demonstrated that potential functions can be used to provide a simple proof of threshold saturation for coupled scalar recursions. In this paper, we present a simple proof of threshold saturation that applies to a wide class of coupled vector recursions. The conditions of the theorem are verified for the density-evolution equations of: (i) joint decoding of irregular LDPC codes for a Slepian-Wolf problem with erasures, (ii) joint decoding of irregular LDPC codes on an erasure multiple-access channel, and (iii) protograph codes on the BEC. This proves threshold saturation for these systems.

Index Terms - convolutional LDPC codes, spatial coupling, threshold saturation, density evolution, potential functions
\end{abstract}

\section{INTRODUCTION}

Low-density parity-check (LDPC) convolutional codes, or spatially-coupled (SC) LDPC codes, were introduced in [1] and observed to have excellent belief-propagation (BP) thresholds in [2], [3], [4]. Recently, they have been observed to approach capacity for a variety of problems [4], [5], [6], [7], [8], [9], [10], [11].

The principle behind their excellent performance is described in [12], where it is shown analytically for the BEC that the BP threshold of a regular SC ensemble converges to the maximum-a-posteriori (MAP) threshold of the uncoupled ensemble. This phenomenon is now called threshold saturation. A similar observation was reported independently in [13] and stated as a conjecture. For binary-input memoryless symmetric (BMS) channels, threshold saturation was empirically observed first [4], [5] and then shown analytically [11].

Threshold saturation now appears to be quite general and spatial-coupling has now been applied, with great success, to more general scenarios in information theory and coding [14], [15], [16], [17], [18], [19], [20], [21]

Recently, the authors introduced a simple proof of threshold saturation for coupled scalar recursions where only a few details must be verified for each system [22]. The examples presented therein prove a number of threshold saturation conjectures made in the aforementioned papers (e.g., see [8],

This material is based upon work supported in part by the National Science Foundation (NSF) under Grants No. 0747470. Any opinions, findings, conclusions, and recommendations expressed in this material are those of the authors and do not necessarily reflect the views of the NSF.
[10]). The proof technique is based on potential functions for density-evolution (DE) recursions and was motivated by the ideas in [23]. Another approach to proving threshold saturation, based on a continuous approximation of spatialcoupling, can be found in [21] and [24].

In this paper, the analysis is extended to prove that threshold saturation also occurs for certain coupled systems of vector recursions. In particular, if the single-system vector recursion can be generated by a scalar potential function, then one can also define a scalar potential function for the coupled system. Using this, one can show that threshold saturation occurs for a wider class of problems. For example, this settles conjectures made in [7], [25]. Along with the results in [26], this shows the universality of SC codes for a noisy SlepianWolf problem with erasures, when the channels are unknown at the transmitter.

\section{A Simple Proof of Threshold Saturation}

In this section, we provide a simple proof of threshold saturation via spatial-coupling for a broad class of vector recursions. The main tool is a potential theory for vector recursions that extends naturally to coupled systems.

\section{A. Notation}

The following notation is used throughout this paper. We let $d \in \mathbb{N}$ be the dimension for the vector recursion, $\mathcal{X} \triangleq$ $[0,1]^{d}$ be the space on which the recursion is defined, and $\mathcal{E} \triangleq[0,1]$ be the parameter space of the recursive system. For convenience, we let $\mathcal{X}_{\circ} \triangleq \mathcal{X} \backslash\{\boldsymbol{0}\}$ and $\mathcal{E}_{\circ} \triangleq \mathcal{E} \backslash\{0\}$. Vectors are denoted in boldface lowercase (e.g. $\boldsymbol{x}, \boldsymbol{y}$ ), are assumed to be row vectors, and inherit the natural partial order $\boldsymbol{x} \preceq \boldsymbol{y}$ defined by $x_{i} \leq y_{i}$ for $1 \leq i \leq d$. Matrices are denoted in boldface capital letters (e.g. $\boldsymbol{X} \in \mathcal{X}^{n}$ ) and we use $\boldsymbol{x}_{i}=[\boldsymbol{X}]_{i}$ to denote the $i$-th row of $\boldsymbol{X}$ and $x_{i, j}=[\boldsymbol{X}]_{i, j}$ to denote the $(i, j)$-th element of $\boldsymbol{X}$. Standardweight typeface is used for scalar-valued functions with a vector/matrix argument (e.g., $F(\boldsymbol{x}), F(\boldsymbol{X})$ ) and boldface is used to denote a vector-valued function with a vector argument (e.g., $\left.\boldsymbol{f}(\boldsymbol{x})=\left[f_{1}(\boldsymbol{x}), \cdots, f_{d}(\boldsymbol{x})\right]\right)$. The gradient of a scalar function is defined by $F^{\prime}(\boldsymbol{x}) \triangleq\left[\partial F(\boldsymbol{x}) / \partial x_{1}, \cdots, \partial F(\boldsymbol{x}) / \partial x_{d}\right]$ and the Jacobian of a vector function is defined by

$$
\boldsymbol{f}^{\prime}(\boldsymbol{x})=\frac{\partial \boldsymbol{f}(\boldsymbol{x})}{\partial \boldsymbol{x}} \triangleq\left[\begin{array}{ccc}
\frac{\partial f_{1}(\boldsymbol{x})}{\partial x_{1}} & \cdots & \frac{\partial f_{1}(\boldsymbol{x})}{\partial x_{d}} \\
\vdots & \ddots & \vdots \\
\frac{\partial f_{d}(\boldsymbol{x})}{\partial x_{1}} & \cdots & \frac{\partial f_{d}(\boldsymbol{x})}{\partial x_{d}}
\end{array}\right]
$$


The gradient of a scalar-valued function with a matrix argument denoted by $F^{\prime}(\boldsymbol{X})$ is a matrix defined by $\left[F^{\prime}(\boldsymbol{X})\right]_{i, j} \triangleq$ $\partial F(\boldsymbol{X}) / \partial x_{i, j}$. For two-argument functionals (e.g., $F(\boldsymbol{x} ; \varepsilon)$ and $U(\boldsymbol{x} ; \varepsilon)$ ), we define $F^{\prime}(\boldsymbol{x} ; \varepsilon) \triangleq \partial F(\boldsymbol{x} ; \varepsilon) / \partial \boldsymbol{x}$.

Abusing notation, we also allow functions defined for vector arguments (e.g., $\boldsymbol{f}(\boldsymbol{x})$ ) to act on matrices (e.g., $\boldsymbol{f}(\boldsymbol{X})$ ) via the rule $[\boldsymbol{f}(\boldsymbol{X})]_{i}=\boldsymbol{f}\left(\boldsymbol{x}_{i}\right)$. The notation $\operatorname{vec}(\boldsymbol{X})$ denotes the transpose of the vector obtained by stacking the columns of $\boldsymbol{X}$ [27]. The Jacobian and Hessian of a matrix function are denoted by

$$
\boldsymbol{f}^{\prime}(\boldsymbol{X}) \triangleq \frac{\partial \operatorname{vec}(\boldsymbol{f}(\boldsymbol{X}))}{\partial \operatorname{vec}(\boldsymbol{X})} \quad \text { and } \quad \boldsymbol{f}^{\prime \prime}(\boldsymbol{x}) \triangleq \frac{\partial \operatorname{vec}\left(\boldsymbol{f}^{\prime}(\boldsymbol{x})\right)}{\partial \boldsymbol{x}} .
$$

\section{B. Single-System Potential}

First, we define potential functions for a class of vector recursions and discuss the associated thresholds.

Definition 1: Let $F: \mathcal{X} \times \mathcal{E} \rightarrow \mathbb{R}$ and $G: \mathcal{X} \rightarrow \mathbb{R}$ be functionals and $\boldsymbol{D}$ be a $d \times d$ positive diagonal matrix. Consider the recursion defined by

$$
\boldsymbol{x}^{(\ell+1)}=\boldsymbol{f}\left(\boldsymbol{g}\left(\boldsymbol{x}^{(\ell)}\right) ; \varepsilon\right),
$$

where $\boldsymbol{f}: \mathcal{X} \times \mathcal{E} \rightarrow \mathcal{X}$ and $\boldsymbol{g}: \mathcal{X} \rightarrow \mathcal{X}$ are mappings defined by $F^{\prime}(\boldsymbol{x} ; \varepsilon)=\boldsymbol{f}(\boldsymbol{x} ; \varepsilon) \boldsymbol{D}$ and $G^{\prime}(\boldsymbol{x})=\boldsymbol{g}(\boldsymbol{x}) \boldsymbol{D}$. Then, the pair $(\boldsymbol{f}, \boldsymbol{g})$ defines a vector admissible system if

i) $\boldsymbol{f}, \boldsymbol{g}$ are twice continuously differentiable,

ii) $\boldsymbol{f}(\boldsymbol{x} ; \varepsilon), \boldsymbol{g}(\boldsymbol{x})$ are non-decreasing in $\boldsymbol{x}$ (w.r.t. $\preceq$ ),

iii) $\boldsymbol{f}(\boldsymbol{x} ; \varepsilon)$ is strictly increasing in $\varepsilon$ for $\boldsymbol{x} \in \mathcal{X}_{\circ}$,

iv) $\boldsymbol{f}(\mathbf{0} ; \varepsilon)=\boldsymbol{f}(\boldsymbol{x} ; 0)=\boldsymbol{g}(\mathbf{0})=\mathbf{0}$ and $F(\boldsymbol{x} ; 0)=0$.

Remark 1: More generally, the vector recursion may have a parameter vector $\varepsilon \in \mathcal{E}^{n}$ and be defined by

$$
\boldsymbol{x}^{(\ell+1)}=\tilde{\boldsymbol{f}}\left(\boldsymbol{g}\left(\boldsymbol{x}^{(\ell)}\right) ; \boldsymbol{\varepsilon}\right) .
$$

In this case, one can consider a path $\varepsilon(\varepsilon) \in \mathcal{E}^{n}$ parameterized by $\varepsilon \in \mathcal{E}$. Such a path is called valid if it is smooth, strictly increasing (w.r.t. the partial order) in $\varepsilon, \varepsilon(0)=\mathbf{0}$, and $\varepsilon(1)=$ 1. The recursion in (2) along a valid path can be converted into a new recursion in the form of (1) with

$$
\boldsymbol{x}^{(\ell+1)}=\boldsymbol{f}\left(\boldsymbol{g}\left(\boldsymbol{x}^{(\ell)}\right) ; \varepsilon\right) \triangleq \tilde{\boldsymbol{f}}\left(\boldsymbol{g}\left(\boldsymbol{x}^{(\ell)}\right) ; \boldsymbol{\varepsilon}(\varepsilon)\right) .
$$

If the resulting functions $(\boldsymbol{f}, \boldsymbol{g})$ satisfy the conditions in Def. 1 for any valid $\varepsilon(\varepsilon)$ path, then the recursion in (2) can be characterized using a scalar $\varepsilon$ analysis and a family of $\varepsilon(\varepsilon)$ paths. This is the approach taken in this work.

Lemma 1: For any $(\boldsymbol{x}, \varepsilon) \in \mathcal{X} \times \mathcal{E}$ and $t \in[0,1]$, we define $\boldsymbol{z}(t) \triangleq \boldsymbol{x}+t(\boldsymbol{f}(\boldsymbol{g}(\boldsymbol{x}) ; \varepsilon)-\boldsymbol{x})$. If $\boldsymbol{x} \preceq \boldsymbol{f}(\boldsymbol{g}(\boldsymbol{x}) ; \varepsilon)$, then $\boldsymbol{z}(t) \preceq$ $\boldsymbol{f}(\boldsymbol{g}(\boldsymbol{z}(t)) ; \varepsilon)$ for all $t \in[0,1]$. Similarly, if $\boldsymbol{x} \succeq \boldsymbol{f}(\boldsymbol{g}(\boldsymbol{x}) ; \varepsilon)$, then $\boldsymbol{z}(t) \succeq \boldsymbol{f}(\boldsymbol{g}(\boldsymbol{z}(t)) ; \varepsilon)$ for all $t \in[0,1]$.

Proof: First, consider the case where $\boldsymbol{x} \preceq \boldsymbol{f}(\boldsymbol{g}(\boldsymbol{x}) ; \varepsilon)$. It is easy to verify that $\boldsymbol{x} \preceq \boldsymbol{z}(t) \preceq \boldsymbol{f}(\boldsymbol{g}(\boldsymbol{x}) ; \varepsilon)$. Since $\boldsymbol{f}$ and $\boldsymbol{g}$ are non-decreasing, one can also observe that

$$
\boldsymbol{z}(t) \preceq \boldsymbol{f}(\boldsymbol{g}(\boldsymbol{x}) ; \varepsilon) \preceq \boldsymbol{f}(\boldsymbol{g}(\boldsymbol{z}(t)) ; \varepsilon) .
$$

Thus, the first claim follows. If $\boldsymbol{x} \succeq \boldsymbol{f}(\boldsymbol{g}(\boldsymbol{x}) ; \varepsilon)$, the same approach shows that $\boldsymbol{z}(t) \succeq \boldsymbol{f}(\boldsymbol{g}(\boldsymbol{x}) ; \varepsilon) \succeq \boldsymbol{f}(\boldsymbol{g}(\boldsymbol{z}(t)) ; \varepsilon)$.
Definition 2: Let the potential function $U(\boldsymbol{x} ; \varepsilon)$ of a vector admissible system $(\boldsymbol{f}, \boldsymbol{g})$ be defined by

$$
\begin{aligned}
U(\boldsymbol{x} ; \varepsilon) & \triangleq \int_{0}^{\boldsymbol{x}}\left[(\boldsymbol{z}-\boldsymbol{f}(\boldsymbol{g}(\boldsymbol{z}) ; \varepsilon)) \boldsymbol{D} \boldsymbol{g}^{\prime}(\boldsymbol{z})\right] \cdot \mathrm{d} \boldsymbol{z} \\
& =\boldsymbol{g}(\boldsymbol{x}) \boldsymbol{D} \boldsymbol{x}^{\top}-G(\boldsymbol{x})-F(\boldsymbol{g}(\boldsymbol{x}) ; \varepsilon),
\end{aligned}
$$

where $U(\mathbf{0} ; \varepsilon)=0$ implies $F(\mathbf{0} ; \varepsilon)=G(\mathbf{0})=0$.

Remark 2: The above result is easily verified by taking the derivative of (3) with respect to $\boldsymbol{x}$. Of course, this also implies that the line integral is path independent. For DE equations, the potential function is related to the pseudo-dual of the Bethe free energy (e.g., see [28, Part 2, pp. 62-65] [29]).

Lemma 2: For any $(\boldsymbol{x}, \varepsilon) \in \mathcal{X} \times \mathcal{E}$, let $\boldsymbol{y} \triangleq \boldsymbol{f}(\boldsymbol{g}(\boldsymbol{x}) ; \varepsilon)$. If $\boldsymbol{x} \preceq \boldsymbol{y}$ or $\boldsymbol{x} \succeq \boldsymbol{y}$, then $U(\boldsymbol{x} ; \varepsilon) \geq U(\boldsymbol{y} ; \varepsilon)$.

Proof: Consider the path from $\boldsymbol{x}$ to $\boldsymbol{y}$ defined by $\boldsymbol{z}(t) \triangleq \boldsymbol{x}+t(\boldsymbol{y}-\boldsymbol{x})$ for $t \in[0,1]$. By Def. 2, one can write

$$
\begin{aligned}
U(\boldsymbol{y} ; \varepsilon) & =U(\boldsymbol{x} ; \varepsilon) \\
& +\int_{0}^{1}\left[(\boldsymbol{z}(t)-\boldsymbol{f}(\boldsymbol{g}(\boldsymbol{z}(t)) ; \varepsilon)) \boldsymbol{D} \boldsymbol{g}^{\prime}(\boldsymbol{z}(t))\right] \cdot \boldsymbol{z}^{\prime}(t) \mathrm{d} t .
\end{aligned}
$$

If $\boldsymbol{x} \preceq \boldsymbol{y}$, then Lem. 1 implies that $\boldsymbol{z}(t)-\boldsymbol{f}(\boldsymbol{g}(\boldsymbol{z}(t)) ; \varepsilon) \preceq \mathbf{0}$ and $\boldsymbol{z}^{\prime}(t) \succeq \mathbf{0}$ for all $t \in[0,1]$. Next, we note that $\boldsymbol{g}(\boldsymbol{x})$ nondecreasing w.r.t. $\preceq$ implies that $\boldsymbol{g}^{\prime}(\boldsymbol{x})$ is a non-negative matrix. Since $\boldsymbol{D}$ and $\boldsymbol{g}^{\prime}(\boldsymbol{x})$ are non-negative matrices and $\boldsymbol{z}^{\prime}(t) \succeq$ $\mathbf{0}$ for $t \in[0,1]$, the integral in (4) is upper bounded by 0 . Similarly, if $\boldsymbol{x} \succeq \boldsymbol{y}$, then $\boldsymbol{z}^{\prime}(t) \preceq \mathbf{0}$ and $\boldsymbol{z}(t)-\boldsymbol{f}(\boldsymbol{g}(\boldsymbol{z}(t)) ; \varepsilon) \succeq$ 0. Again, this implies the integral in (4) is upper bounded by 0 . Therefore, we conclude that $U(\boldsymbol{y} ; \varepsilon) \leq U(\boldsymbol{x} ; \varepsilon)$.

Definition 3: For $\boldsymbol{x} \in \mathcal{X}$ and $\varepsilon \in \mathcal{E}$,

i) $\boldsymbol{x}$ is a fixed point (f.p.) if $\boldsymbol{x}=\boldsymbol{f}(\boldsymbol{g}(\boldsymbol{x}) ; \varepsilon)$.

ii) $\boldsymbol{x}$ is a stationary point (s.p.) if $U^{\prime}(\boldsymbol{x} ; \varepsilon)=\mathbf{0}$.

Definition 4: The fixed-point set, $\mathcal{F}(\varepsilon)$, its $x$-support, $\mathcal{X}_{f}$, and the epsilon set, $\varepsilon(\boldsymbol{x})$, are given by

$$
\begin{aligned}
\mathcal{F}(\varepsilon) & \triangleq\left\{\boldsymbol{x} \in \mathcal{X}_{\circ} \mid \boldsymbol{x}=\boldsymbol{f}(\boldsymbol{g}(\boldsymbol{x}) ; \varepsilon)\right\} \\
\mathcal{X}_{f} & \triangleq \cup_{\varepsilon \in \mathcal{E}} \mathcal{F}(\varepsilon) \\
\varepsilon(\boldsymbol{x}) & \triangleq\{\varepsilon \in \mathcal{E} \mid \boldsymbol{x} \in \mathcal{F}(\varepsilon)\} .
\end{aligned}
$$

Lemma 3: For a vector admissible system, we have

i) $U(\boldsymbol{x} ; \varepsilon)$ is strictly decreasing in $\varepsilon$, for $\boldsymbol{x} \in \mathcal{X}_{\circ}$ and $\varepsilon \in \mathcal{E}$.

ii) Any f.p. $\boldsymbol{x} \in \mathcal{X}$ is a s.p. of the potential.

iii) For any $x \in \mathcal{X}_{f}, \varepsilon(\boldsymbol{x})$ has a single element.

Proof: The potential function is a line integral of $(\boldsymbol{z}-\boldsymbol{f}(\boldsymbol{g}(\boldsymbol{z}) ; \varepsilon)) \boldsymbol{D} \boldsymbol{g}^{\prime}(\boldsymbol{z})$, and this expression is strictly decreasing in $\varepsilon$, for $\boldsymbol{x} \in \mathcal{X}_{\circ}$ and $\varepsilon \in \mathcal{E}$. The second property also follows from this formulation because, at a f.p., the integrand is $\mathbf{0}$. For the third, let $\boldsymbol{x} \in \mathcal{X}_{f}$ and observe that the definition of $\mathcal{X}_{f}$ implies $\varepsilon(\boldsymbol{x})$ has at least one element. Since $\boldsymbol{f}(\boldsymbol{x} ; \varepsilon)$ is strictly increasing in $\varepsilon$, for $\boldsymbol{x} \in \mathcal{X}_{\circ}$ and $\varepsilon \in \mathcal{E}$, it follows that $\boldsymbol{x}=\boldsymbol{f}(\boldsymbol{g}(\boldsymbol{x}) ; \varepsilon)$ can have at most one $\varepsilon$-root. Therefore, $\varepsilon(\boldsymbol{x})$ is a singleton and we treat it as a function $\varepsilon: \mathcal{X}_{f} \rightarrow \mathcal{E}$.

Definition 5: For a pair $(\boldsymbol{x}, \varepsilon) \in \mathcal{X} \times \mathcal{E}$, let $\boldsymbol{x}^{(0)}=\boldsymbol{x}$ be the initial value of the recursion defined in (1). We define $\boldsymbol{x}^{\infty}(\boldsymbol{x} ; \varepsilon)=\lim _{\ell \rightarrow \infty} \boldsymbol{f}\left(\boldsymbol{g}\left(\boldsymbol{x}^{(\ell)}\right) ; \varepsilon\right)$ if the limit exits. 
Lemma 4: For any $(\boldsymbol{x}, \varepsilon) \in \mathcal{X} \times \mathcal{E}$, if $\boldsymbol{x} \preceq \boldsymbol{f}(\boldsymbol{g}(\boldsymbol{x}) ; \varepsilon)$ or $\boldsymbol{x} \succeq \boldsymbol{f}(\boldsymbol{g}(\boldsymbol{x}) ; \varepsilon)$, then $\boldsymbol{x}^{\infty}(\boldsymbol{x} ; \varepsilon)$ exists and

$$
U(\boldsymbol{x} ; \varepsilon) \geq U\left(\boldsymbol{x}^{\infty}(\boldsymbol{x} ; \varepsilon) ; \varepsilon\right) .
$$

Proof: Consider the case where $\boldsymbol{x} \preceq \boldsymbol{f}(\boldsymbol{g}(\boldsymbol{x}) ; \varepsilon)$, and let $\boldsymbol{x}^{(0)}=\boldsymbol{x}$. By the fact that $\boldsymbol{x}^{(1)}=\boldsymbol{f}(\boldsymbol{g}(\boldsymbol{x}) ; \varepsilon) \succeq \boldsymbol{x}^{(0)}$, the sequence of the vectors $\boldsymbol{x}^{(\ell)}$ for $\ell \geq 0$ satisfies $\boldsymbol{x}^{(0)} \preceq \boldsymbol{x}^{(1)} \preceq$ $\boldsymbol{x}^{(2)} \preceq \cdots \preceq \mathbf{1}$. This implies that, for $i=1, \ldots, d,\left[\boldsymbol{x}^{(\ell)}\right]_{i}$ is a non-decreasing real sequence upper bounded by 1 . Therefore, the limit $\boldsymbol{x}^{\infty}(\boldsymbol{x} ; \varepsilon)$ exists. Similarly, if $\boldsymbol{x} \succeq \boldsymbol{f}(\boldsymbol{g}(\boldsymbol{x}) ; \varepsilon)$, then the limit exists because each $\left[\boldsymbol{x}^{(\ell)}\right]_{i}$ is non-increasing and lower bounded by 0 . Finally, Lem. 2 implies that, for all $\ell \geq 0, U\left(\boldsymbol{x}^{(\ell)} ; \varepsilon\right) \geq U\left(\boldsymbol{x}^{(\ell+1)} ; \varepsilon\right)$. Therefore, the inequality (5) follows.

Corollary 1: For all $\varepsilon \in \mathcal{E}, \boldsymbol{x}^{\infty}(\mathbf{1} ; \varepsilon)$ exists.

Proof: This follows from $\mathbf{1} \succeq \boldsymbol{f}(\boldsymbol{g}(\mathbf{1}) ; \varepsilon)$ for $\varepsilon \in \mathcal{E}$.

Definition 6: The single-system threshold is defined to be

$$
\varepsilon_{s}^{*} \triangleq \sup \left\{\varepsilon \in \mathcal{E} \mid \boldsymbol{x}^{\infty}(\mathbf{1} ; \varepsilon)=\mathbf{0}\right\},
$$

and is the $\varepsilon$-threshold for convergence of the recursion to $\mathbf{0}$.

Remark 3: The recursion (1) has no f.p.s in $\mathcal{X}_{\circ}$ iff $\varepsilon<\varepsilon_{s}^{*}$. For DE recursions associated with BP decoding, the threshold $\varepsilon_{s}^{*}$ is called the BP threshold.

Definition 7: The potential threshold is defined by

$$
\varepsilon^{*} \triangleq \sup \left\{\varepsilon \in \mathcal{E} \mid \min _{\boldsymbol{x} \in \mathcal{F}(\varepsilon)} U(\boldsymbol{x} ; \varepsilon) \geq 0\right\} .
$$

This is well defined because $U(\boldsymbol{x} ; 0) \geq 0$. For $\varepsilon>\varepsilon_{s}^{*}$, the quantity $\Delta E(\varepsilon) \triangleq \min _{\boldsymbol{x} \in \mathcal{F}(\varepsilon)} U(\boldsymbol{x} ; \varepsilon)$ is the called energy gap and $\varepsilon_{s}^{*}<\varepsilon<\varepsilon^{*}$ implies $\Delta E(\varepsilon)>0$. Since $U(\boldsymbol{x} ; \varepsilon)$ is strictly decreasing in $\varepsilon$, $\varepsilon^{*} \leq \varepsilon^{\prime}$ for any $\varepsilon^{\prime}$ that satisfies $\Delta E\left(\varepsilon^{\prime}\right)=0$.

Definition 8: The fixed-point potential, $Q: \mathcal{X}_{f} \rightarrow \mathbb{R}$, is defined by $Q(\boldsymbol{x})=U(\boldsymbol{x} ; \varepsilon(\boldsymbol{x}))$. The potential threshold defined in (6) can be rewritten as

$$
\varepsilon^{*} \triangleq \sup \left\{\varepsilon \in \mathcal{E} \mid \min _{\boldsymbol{x} \in \mathcal{F}(\varepsilon)} Q(\boldsymbol{x}) \geq 0\right\} .
$$

Remark 4: Consider the fixed points $\boldsymbol{x}_{1}, \boldsymbol{x}_{2} \in \mathcal{X}_{f}$ and observe that $Q\left(\boldsymbol{x}_{1}\right)=U\left(\boldsymbol{x}_{1} ; \varepsilon\left(\boldsymbol{x}_{1}\right)\right)$ and $Q\left(\boldsymbol{x}_{2}\right)=U\left(\boldsymbol{x}_{2} ; \varepsilon\left(\boldsymbol{x}_{2}\right)\right)$. Suppose there is a differentiable path $\boldsymbol{z}(t) \in \mathcal{X}_{f}$ from $\boldsymbol{x}_{1}$ to $\boldsymbol{x}_{2}$ such that $\varepsilon(\boldsymbol{z}(t))$ is differentiable for $t \in[0,1]$. Then, $Q^{\prime}(\boldsymbol{x})$ exists and

$$
\begin{aligned}
Q\left(\boldsymbol{x}_{2}\right) & =Q\left(\boldsymbol{x}_{1}\right)+\int_{\boldsymbol{x}_{1}}^{\boldsymbol{x}_{2}} Q^{\prime}(\boldsymbol{z}) \cdot \mathrm{d} \boldsymbol{z} \\
& =Q\left(\boldsymbol{x}_{1}\right)+\int_{0}^{1} U^{(0,1)}(\boldsymbol{z}(t) ; \varepsilon(\boldsymbol{z}(t))) \varepsilon^{\prime}(\boldsymbol{z}(t)) \cdot \boldsymbol{z}^{\prime}(t) \mathrm{d} t .
\end{aligned}
$$

The following lemma compares the minimum fixed-point potentials of two different $\varepsilon$ parameters.

Lemma 5: Let $\varepsilon_{1}, \varepsilon_{2} \in \mathcal{E}$ satisfy $\varepsilon_{1}<\varepsilon_{2}, \mathcal{F}\left(\varepsilon_{1}\right) \neq \emptyset$, and $\mathcal{F}\left(\varepsilon_{2}\right) \neq \emptyset$. Then,

$$
\min _{\boldsymbol{x} \in \mathcal{F}\left(\varepsilon_{1}\right)} Q(\boldsymbol{x})>\min _{\boldsymbol{x} \in \mathcal{F}\left(\varepsilon_{2}\right)} Q(\boldsymbol{x}) .
$$

Proof: Let $\boldsymbol{x}_{1} \in \mathcal{F}\left(\varepsilon_{1}\right)$ and $\boldsymbol{x}_{2} \in \mathcal{F}\left(\varepsilon_{2}\right)$ be the fixed points that achieve the minimums in (9). Since $\boldsymbol{f}(\boldsymbol{x} ; \varepsilon)$ is strictly increasing in $\varepsilon$, one finds that

$$
\boldsymbol{x}_{1}=\boldsymbol{f}\left(\boldsymbol{g}\left(\boldsymbol{x}_{1}\right) ; \varepsilon_{1}\right) \preceq \boldsymbol{f}\left(\boldsymbol{g}\left(\boldsymbol{x}_{1}\right) ; \varepsilon_{2}\right) \preceq \boldsymbol{x}^{\infty}\left(\boldsymbol{x}_{1} ; \varepsilon_{2}\right) .
$$

Using $\boldsymbol{x}^{\infty}\left(\boldsymbol{x}_{1} ; \varepsilon_{2}\right) \in \mathcal{F}\left(\varepsilon_{2}\right)$, the proof concludes with

$$
\begin{aligned}
\min _{\boldsymbol{x} \in \mathcal{F}\left(\varepsilon_{1}\right)} Q(\boldsymbol{x}) & =U\left(\boldsymbol{x}_{1} ; \varepsilon_{1}\right) \stackrel{(a)}{>} U\left(\boldsymbol{x}_{1} ; \varepsilon_{2}\right) \stackrel{(b)}{\geq} U\left(\boldsymbol{x}^{\infty}\left(\boldsymbol{x}_{1} ; \varepsilon_{2}\right) ; \varepsilon_{2}\right) \\
& \geq \min _{\boldsymbol{x} \in \mathcal{F}\left(\varepsilon_{2}\right)} U\left(\boldsymbol{x} ; \varepsilon_{2}\right)=\min _{\boldsymbol{x} \in \mathcal{F}\left(\varepsilon_{2}\right)} Q(\boldsymbol{x}),
\end{aligned}
$$

where the inequality $(a)$ is from Lem. 3, and the inequality (b) is the result of Lem. 4.

Remark 5: Since $\min _{\boldsymbol{x} \in \mathcal{F}(\varepsilon)} Q(\boldsymbol{x})$ is strictly decreasing in $\varepsilon$, one finds $U(\boldsymbol{x} ; \varepsilon)>0$ for all $\boldsymbol{x} \in \mathcal{F}(\varepsilon)$ when $\varepsilon \in\left(\varepsilon_{s}^{*}, \varepsilon^{*}\right)$. If $\varepsilon^{*}<\varepsilon \leq 1$, then one also has $\min _{\boldsymbol{x} \in \mathcal{F}(\varepsilon)} Q(\boldsymbol{x})<0$.

\section{Coupled-System Potential}

We now extend our definition of potential functions to coupled systems of vector recursions. In particular, we consider a "spatial-coupling" of the single system recursion, (1), that gives rise to the recursion (10) and a closely related matrix recursion (11). For the matrix recursion of the coupled system, we define a potential function and show that, for $\varepsilon<\varepsilon^{*}$, the only fixed point of the coupled system is the zero matrix. We note that a similar potential was defined earlier for the special case of a scalar Curie-Weiss system in [15].

Definition 9 (cf. [12]): The basic spatially-coupled vector system is defined by placing $2 L+1 \boldsymbol{f}$-systems at positions in the set $\mathcal{L}_{f}=\{-L,-L+1, \ldots, L\}$ and coupling them with $2 L+w \boldsymbol{g}$-systems at positions in the set $\mathcal{L}_{\boldsymbol{g}}=\{-L,-L+$ $1, \ldots, L+(w-1)\}$. For the coupled system, this leads to the recursion, for $i \in \mathcal{L}_{\boldsymbol{g}}$, given by

$$
\boldsymbol{x}_{i}^{(\ell+1)}=\frac{1}{w} \sum_{k=0}^{w-1} \boldsymbol{f}\left(\frac{1}{w} \sum_{j=0}^{w-1} \boldsymbol{g}\left(\boldsymbol{x}_{i+j-k}^{(\ell)}\right) ; \varepsilon_{i-k}\right),
$$

where $\varepsilon_{i}=\varepsilon$ for $i \in \mathcal{L}_{\boldsymbol{f}}, \varepsilon_{i}=0$ for $i \notin \mathcal{L}_{\boldsymbol{f}}, \boldsymbol{x}_{i}^{(0)}=\mathbf{1}$ for $i \in \mathcal{L}_{\boldsymbol{g}}$, and $\boldsymbol{x}_{i}^{(\ell)}=\mathbf{0}$ for $i \notin \mathcal{L}_{\boldsymbol{g}}$ and all $\ell$.

Definition 10 (cf. [22]): The recursion in (10) can be rewritten as a matrix recursion. Let $\boldsymbol{X} \in \mathcal{X}^{2 L+w}$ have the decomposition $\boldsymbol{X}=\left[\boldsymbol{x}_{-L}^{\top}, \cdots, \boldsymbol{x}_{L+w-1}^{\top}\right]^{\top}$. Then, (10) is given by

$$
\boldsymbol{X}^{(\ell+1)}=\boldsymbol{A}^{\top} \boldsymbol{f}\left(\boldsymbol{A g}\left(\boldsymbol{X}^{(\ell)}\right) ; \varepsilon\right),
$$

where $\boldsymbol{A}$ is the following $(2 L+1) \times(2 L+w)$ matrix.

$$
\left.\boldsymbol{A}=\frac{1}{w}\left[\begin{array}{cccccccc}
\overbrace{1} & 1 & \cdots & 1 & 0 & 0 & \cdots & 0 \\
0 & 1 & 1 & \cdots & 1 & 0 & \cdots & 0 \\
\vdots & \ddots & \ddots & \ddots & \ddots & \ddots & \ddots & \vdots \\
0 & \cdots & 0 & 1 & 1 & \cdots & 1 & 0 \\
0 & \cdots & 0 & 0 & 1 & 1 & \cdots & 1
\end{array}\right]\right\} \underset{+}{+}
$$

Definition 11 (cf. [12]): Let $i_{0} \triangleq\left\lfloor\frac{w-1}{2}\right\rfloor$. The one-sided spatially-coupled vector system is a modification of (10) defined by fixing the values of positions outside $\mathcal{L}_{f}^{\prime}=$ $\left\{-L, \ldots, i_{0}\right\}$. It forces the remaining values to $\boldsymbol{x}_{i_{0}}^{(\ell)}$, that is $\boldsymbol{x}_{i}^{(\ell)}=\boldsymbol{x}_{i_{0}}^{(\ell)}$ for $i_{0}<i \leq 2 L+(w-1)$ and all $\ell$. 
Lemma 6 (cf. [12, Lem. 14]): For both the basic and onesided SC systems, the recursions are componentwise decreasing with iteration and converge to well-defined fixed points. The one-sided recursion in Def. 11 is a componentwise upper bound on the basic SC recursion for $i \in \mathcal{L}_{g}$ and converges to a non-decreasing fixed-point vector.

Sketch of Proof: The proof follows from the monotonicity of $\boldsymbol{f}, \boldsymbol{g}$ and a careful treatment of the boundary conditions.

Definition 12: The coupled-system potential for admissible matrix recursions is defined to be

$$
U(\boldsymbol{X} ; \varepsilon) \triangleq \operatorname{Tr}\left(\boldsymbol{g}(\boldsymbol{X}) \boldsymbol{D} \boldsymbol{X}^{\top}\right)-G(\boldsymbol{X})-F(\boldsymbol{A g}(\boldsymbol{X}) ; \varepsilon),
$$

where $G(\boldsymbol{X})=\sum_{i} G\left(\boldsymbol{x}_{i}\right)$ and $F(\boldsymbol{X} ; \varepsilon)=\sum_{i} F\left(\boldsymbol{x}_{i} ; \varepsilon\right)$.

Remark 6: The key property of the above coupled-system potential is that the derivative w.r.t. $[\boldsymbol{X}]_{i}=\boldsymbol{x}_{i}$ has the form

$$
\left[U^{\prime}(\boldsymbol{X} ; \varepsilon)\right]_{i}=\left(\boldsymbol{x}_{i}-\left[\boldsymbol{A}^{\top} \boldsymbol{f}(\boldsymbol{A g}(\boldsymbol{X}) ; \varepsilon)\right]_{i}\right) \boldsymbol{D} \boldsymbol{g}^{\prime}\left(\boldsymbol{x}_{i}\right) .
$$

To see this, one can compute the derivative of each term separately. For the first term, we have

$$
\begin{aligned}
\frac{\partial}{\partial \boldsymbol{x}_{i}} \operatorname{Tr}\left(\boldsymbol{g}(\boldsymbol{X}) \boldsymbol{D} \boldsymbol{X}^{\top}\right) & =\frac{\partial}{\partial \boldsymbol{x}_{i}} \sum_{j=-L}^{L+w-1} \boldsymbol{g}\left(\boldsymbol{x}_{j}\right) \boldsymbol{D} \boldsymbol{x}_{j}^{\top} \\
& =\boldsymbol{x}_{i} \boldsymbol{D} \boldsymbol{g}^{\prime}\left(\boldsymbol{x}_{i}\right)+\boldsymbol{g}\left(\boldsymbol{x}_{i}\right) \boldsymbol{D} .
\end{aligned}
$$

For the second term, we use $\left(\partial / \partial \boldsymbol{x}_{i}\right) G(\boldsymbol{X})=\boldsymbol{g}\left(\boldsymbol{x}_{i}\right) \boldsymbol{D}$. For the third term, we use

$$
\begin{array}{r}
\frac{\partial}{\partial \boldsymbol{x}_{i}} F(\boldsymbol{A g}(\boldsymbol{X}) ; \varepsilon)=\frac{\partial}{\partial \boldsymbol{x}_{i}} \sum_{j=-L}^{L} F\left(\sum_{k=-L}^{L+w-1} a_{j, k} \boldsymbol{g}\left(\boldsymbol{x}_{k}\right) ; \varepsilon\right) \\
=\sum_{j=-L}^{L} a_{j, i} \boldsymbol{f}\left(\sum_{k=-L}^{L+w-1} a_{j, k} \boldsymbol{g}\left(\boldsymbol{x}_{k}\right) ; \varepsilon\right) \boldsymbol{D} \boldsymbol{g}^{\prime}\left(\boldsymbol{x}_{i}\right) \\
=\left[\boldsymbol{A}^{\top} \boldsymbol{f}(\boldsymbol{A} \boldsymbol{g}(\boldsymbol{X}) ; \varepsilon)\right]_{i} \boldsymbol{D} \boldsymbol{g}^{\prime}\left(\boldsymbol{x}_{i}\right) .
\end{array}
$$

Definition 13: The down-shift operator $\boldsymbol{S}_{n}: \mathcal{X}^{n} \rightarrow \mathcal{X}^{n}$ is defined by $\left[\boldsymbol{S}_{n} \boldsymbol{X}\right]_{1}=\mathbf{0}$ and $\left[\boldsymbol{S}_{n} \boldsymbol{X}\right]_{i}=\boldsymbol{x}_{i-1}$ for $2 \leq i \leq n$. In the sequel, the subscript of the down-shift operator is omitted, and it can be inferred from the context.

Lemma 7: Let $\boldsymbol{X} \in \mathcal{X}^{n}$ be a matrix with non-decreasing columns generated by averaging the rows of $\boldsymbol{Z} \in \mathcal{X}^{n}$ over a sliding window of size $w$. Then, $\|\operatorname{vec}(\boldsymbol{S} \boldsymbol{X}-\boldsymbol{X})\|_{\infty} \leq \frac{1}{w}$ and $\|\operatorname{vec}(\boldsymbol{S} \boldsymbol{X}-\boldsymbol{X})\|_{1}=\left\|\boldsymbol{x}_{n}\right\|_{1}=\|\boldsymbol{X}\|_{\infty}$.

Proof: For $\|\operatorname{vec}(\boldsymbol{S} \boldsymbol{X}-\boldsymbol{X})\|_{\infty}$, one has

$$
\left|x_{i, j}-x_{i-1, j}\right|=\left|\frac{1}{w} \sum_{k=0}^{w-1} z_{i+k, j}-\frac{1}{w} \sum_{k=0}^{w-1} z_{i-1+k, j}\right| \leq \frac{1}{w} .
$$

Since the columns of $\boldsymbol{X}$ are non-decreasing, the 1-norm sum telescopes and we get $\|\operatorname{vec}(\boldsymbol{S} \boldsymbol{X}-\boldsymbol{X})\|_{1}=\left\|\boldsymbol{x}_{n}\right\|_{1}=\|\boldsymbol{X}\|_{\infty}$.

Lemma 8: Let $\boldsymbol{X} \in \mathcal{X}^{2 L+w}$ have the decomposition $\boldsymbol{X}=$ $\left[\boldsymbol{x}_{-L}^{\top}, \cdots, \boldsymbol{x}_{L+w-1}^{\top}\right]^{\top}$ and satisfy $[\boldsymbol{X}]_{i}=[\boldsymbol{X}]_{i_{0}}$ for $i>i_{0}$. Then, $U(\boldsymbol{S} \boldsymbol{X} ; \varepsilon)-U(\boldsymbol{X} ; \varepsilon) \leq-U\left(\boldsymbol{x}_{i_{0}} ; \varepsilon\right)$.

Proof: First, we rewrite the potential as the summation

$$
U(\boldsymbol{X} ; \varepsilon)=\sum_{i=-L}^{L+w-1} \boldsymbol{g}\left(\boldsymbol{x}_{i}\right) \boldsymbol{D} \boldsymbol{x}_{i}^{\top}-G\left(\boldsymbol{x}_{i}\right)-\sum_{i=-L}^{L} F\left([\boldsymbol{A g}(\boldsymbol{X})]_{i} ; \varepsilon\right) \text {. }
$$

Next, we compute $U(\boldsymbol{S} \boldsymbol{X} ; \varepsilon)-U(\boldsymbol{X} ; \varepsilon)$ separately each of the three terms in $U(\boldsymbol{X} ; \varepsilon)$. The first term, $T_{1}$, equals

$$
\begin{aligned}
T_{1} & =\sum_{i=-L+1}^{L+w-2} \boldsymbol{g}\left(\boldsymbol{x}_{i}\right) \boldsymbol{D} \boldsymbol{x}_{i}^{\top}-\sum_{i=-L}^{L+w-1} \boldsymbol{g}\left(\boldsymbol{x}_{i}\right) \boldsymbol{D} \boldsymbol{x}_{i}^{\top} \\
& =-\boldsymbol{g}\left(\boldsymbol{x}_{L+w-1}\right) \boldsymbol{D} \boldsymbol{x}_{L+w-1}^{\top}=-\boldsymbol{g}\left(\boldsymbol{x}_{i_{0}}\right) \boldsymbol{D} \boldsymbol{x}_{i_{0}}^{\top} .
\end{aligned}
$$

Similarly, the second term is given by $T_{2}=G\left(\boldsymbol{x}_{i_{0}}\right)$. For the third term, it follows from $F(\boldsymbol{x} ; \varepsilon) \geq 0$ and $[\boldsymbol{A g}(\boldsymbol{S} \boldsymbol{X})]_{i}=$ $[\boldsymbol{A g}(\boldsymbol{X})]_{i-1}$ (for $i \in \mathcal{L}_{\boldsymbol{g}} \backslash\{-L\}$ ), that

$$
-F(\boldsymbol{A S} \boldsymbol{g}(\boldsymbol{X}) ; \varepsilon) \leq-\sum_{i=-L}^{L-1} F\left([\boldsymbol{A g}(\boldsymbol{X})]_{i} ; \varepsilon\right) .
$$

Therefore, the third term satisfies

$$
\begin{aligned}
T_{3} & =-F(\boldsymbol{A S} \boldsymbol{g}(\boldsymbol{X}) ; \varepsilon)+F(\boldsymbol{A g}(\boldsymbol{X}) ; \varepsilon) \\
& \leq-\sum_{i=-L}^{L-1} F\left([\boldsymbol{A g}(\boldsymbol{X})]_{i} ; \varepsilon\right)+\sum_{i=-L}^{L} F\left([\boldsymbol{A g}(\boldsymbol{X})]_{i} ; \varepsilon\right) \\
& =F\left([\boldsymbol{A g}(\boldsymbol{X})]_{L} ; \varepsilon\right)=F\left(\boldsymbol{g}\left(\boldsymbol{x}_{i_{0}}\right) ; \varepsilon\right) .
\end{aligned}
$$

Finally, we can write

$$
\begin{aligned}
& U(\boldsymbol{S} \boldsymbol{X} ; \varepsilon)-U(\boldsymbol{X} ; \varepsilon)=T_{1}+T_{2}+T_{3} \leq \\
& \quad \boldsymbol{g}\left(\boldsymbol{x}_{i_{0}}\right) \boldsymbol{D} \boldsymbol{x}_{i_{0}}^{\top}+G\left(\boldsymbol{x}_{i_{0}}\right)+F\left(\boldsymbol{g}\left(\boldsymbol{x}_{i_{0}}\right) ; \varepsilon\right)=-U\left(\boldsymbol{x}_{i_{0}} ; \varepsilon\right) .
\end{aligned}
$$

Lemma 9: For a fixed point of the one-sided SC system $\boldsymbol{X}$,

$$
\operatorname{vec}\left(U^{\prime}(\boldsymbol{X} ; \varepsilon)\right) \cdot \operatorname{vec}(\boldsymbol{S} \boldsymbol{X}-\boldsymbol{X})=\mathbf{0} .
$$

Proof: Rows $1, \ldots, i_{0}+L$ of $U^{\prime}(\boldsymbol{X} ; \varepsilon)$ are zero since $\boldsymbol{X}$ is a fixed point of the one-sided spatially-coupled system. Also, rows $L+i_{0}+1, \ldots, 2 L+(w-1)$ of $\boldsymbol{S} \boldsymbol{X}-\boldsymbol{X}$ are all-zero due to the right boundary constraint. Hence, the inner product of these two terms is identically zero.

Lemma 10: Let $\boldsymbol{X}$ be a fixed point of the one-sided SC system. Then, $\boldsymbol{x}^{\infty}\left(\boldsymbol{x}_{i_{0}} ; \varepsilon\right)$ exists, $\boldsymbol{x}_{i_{0}} \preceq \boldsymbol{x}^{\infty}\left(\boldsymbol{x}_{i_{0}} ; \varepsilon\right)$, and $U\left(\boldsymbol{x}_{i_{0}} ; \varepsilon\right) \geq U\left(\boldsymbol{x}^{\infty}\left(\boldsymbol{x}_{i_{0}} ; \varepsilon\right) ; \varepsilon\right)$.

Proof: From Lem. 6, $\boldsymbol{X}$ is non-decreasing. From (10) and by the fact that $\boldsymbol{X}$ is a fixed point of the one-sided SC system, it can be shown that

$$
\begin{aligned}
\boldsymbol{x}_{i_{0}} & =\frac{1}{w} \sum_{k=0}^{w-1} \boldsymbol{f}\left(\frac{1}{w} \sum_{j=0}^{w-1} \boldsymbol{g}\left(\boldsymbol{x}_{i_{0}+j-k}\right) ; \varepsilon\right) \\
& \preceq \frac{1}{w} \sum_{k=0}^{w-1} \boldsymbol{f}\left(\frac{1}{w} \sum_{j=0}^{w-1} \boldsymbol{g}\left(\boldsymbol{x}_{i_{0}}\right) ; \varepsilon\right)=\boldsymbol{f}\left(\boldsymbol{g}\left(\boldsymbol{x}_{i_{0}}\right) ; \varepsilon\right) .
\end{aligned}
$$

By applying Lem. 4, the claim of the lemma follows.

Lemma 11: The norm of the Hessian, $U^{\prime \prime}(\boldsymbol{X} ; \varepsilon)$, of the SC potential is bounded by a constant independent of $L$ and $w$.

Proof: By direct computation, we obtain

$$
\left\|U^{\prime \prime}(\boldsymbol{X} ; \varepsilon)\right\|_{\infty} \leq\|\boldsymbol{D}\|_{\infty}\left(g_{m}^{\prime}+g_{m}^{\prime \prime}+2\left(g_{m}^{\prime}\right)^{2} f_{m}^{\prime}\right) \triangleq K_{\boldsymbol{f}, \boldsymbol{g}},
$$

where $g_{m}^{\prime}=\sup _{\boldsymbol{x} \in \mathcal{X}}\left\|\boldsymbol{g}^{\prime}(\boldsymbol{x})\right\|_{\infty}, g_{m}^{\prime \prime}=\sup _{\boldsymbol{x} \in \mathcal{X}}\left\|\boldsymbol{g}^{\prime \prime}(\boldsymbol{x})\right\|_{\infty}$ and $f_{m}^{\prime}=\sup _{\boldsymbol{x} \in \mathcal{X}}\left\|\boldsymbol{f}^{\prime}(\boldsymbol{x} ; \varepsilon)\right\|_{\infty}$. 
Theorem 1: For a vector admissible system $(\boldsymbol{f}, \boldsymbol{g})$ with $\varepsilon<$ $\varepsilon^{*}$ and $w>\left(d K_{\boldsymbol{f}, \boldsymbol{g}}\right) /(2 \Delta E(\varepsilon))$, the only fixed point of the spatially-coupled system (Def. 10) is $\mathbf{0 .}$

Proof: Fix $\varepsilon<\varepsilon^{*}$ and $w>\left(d K_{\boldsymbol{f}, \boldsymbol{g}}\right) /(2 \Delta E(\varepsilon))$. Suppose $\boldsymbol{X} \neq \boldsymbol{0}$ is the unique fixed point (Lem. 6) of the one-sided recursion in Def. 11. Using Taylor's Theorem, the secondorder expansion of $U(\boldsymbol{S} \boldsymbol{X} ; \varepsilon)$ about $\boldsymbol{X}$ gives

$$
\begin{array}{ll}
\frac{1}{2} \operatorname{vec}(\boldsymbol{S} \boldsymbol{X}-\boldsymbol{X})^{\top} U^{\prime \prime}(\boldsymbol{Z}(t) ; \varepsilon) \operatorname{vec}(\boldsymbol{S} \boldsymbol{X}-\boldsymbol{X}) \\
=U(\boldsymbol{S} \boldsymbol{X} ; \varepsilon)-U(\boldsymbol{X} ; \varepsilon)-\operatorname{vec}\left(U^{\prime}(\boldsymbol{X} ; \varepsilon)\right) \cdot \operatorname{vec}(\boldsymbol{S} \boldsymbol{X}-\boldsymbol{X}) \\
=U(\boldsymbol{S} \boldsymbol{X} ; \varepsilon)-U(\boldsymbol{X} ; \varepsilon) & (\text { Lem. 9) } \\
\leq-U\left(\boldsymbol{x}_{i_{0}} ; \varepsilon\right) & (\text { Lem. 8 }) \\
\leq-U\left(\boldsymbol{x}^{(\infty)}\left(\boldsymbol{x}_{i_{0}} ; \varepsilon\right) ; \varepsilon\right) & (\text { Lem. 10 ) } \\
\leq-\Delta E(\varepsilon), & (\text { Def. 7 ) }
\end{array}
$$

for some $t \in[0,1]$ with $\boldsymbol{Z}(t)=\boldsymbol{X}+t(\boldsymbol{S} \boldsymbol{X}-\boldsymbol{X})$. Taking the absolute value and using Lemmas 7 and 11 gives

$$
\begin{aligned}
& \Delta E(\varepsilon) \leq\left|\frac{1}{2} \operatorname{vec}(\boldsymbol{S} \boldsymbol{X}-\boldsymbol{X})^{T} U^{\prime \prime}(\boldsymbol{Z}(t) ; \varepsilon) \operatorname{vec}(\boldsymbol{S} \boldsymbol{X}-\boldsymbol{X})\right| \\
& \quad \leq \frac{1}{2}\|\operatorname{vec}(\boldsymbol{S} \boldsymbol{X}-\boldsymbol{X})\|_{1}\left\|U^{\prime \prime}(\boldsymbol{Z}(t) ; \varepsilon)\right\|_{\infty}\|\operatorname{vec}(\boldsymbol{S} \boldsymbol{X}-\boldsymbol{X})\|_{\infty} \\
& \quad \leq \frac{1}{2}\|\boldsymbol{X}\|_{\infty} K_{\boldsymbol{f}, \boldsymbol{g}} \frac{1}{w} \leq \frac{d}{2 w} K_{\boldsymbol{f}, \boldsymbol{g} .}
\end{aligned}
$$

This implies that $w \leq\left(d K_{\boldsymbol{f}, \boldsymbol{g}}\right) /(2 \Delta E(\varepsilon))$, but that contradicts the hypothesis. Thus, the only fixed point for the onesided spatially-coupled system is the trivial fixed point $\mathbf{0}$. Also, the one-sided spatially-coupled system upper bounds the twosided system. Hence, the only fixed point of the two-sided system is $\mathbf{0}$.

\section{Applications}

In this section, we apply Theorem 1 to a few coding problems that have vector DE recursions. To save space, we rely on definitions and notation from [30], [31], [32].

\section{A. Potential Function and EBP EXIT Curves}

First, we introduce a connection between the single-system potential function and the EBP EXIT curves used in [26]. As discussed in Rem. 1, the potential function can be constructed along a smooth and strictly increasing (w.r.t. the partial order) path $\varepsilon(\varepsilon)$ parameterized by $\varepsilon \in \mathcal{E}$. Given a parameter threshold $\varepsilon^{\prime}$ computed from the potential function, the corresponding vector threshold $\varepsilon\left(\varepsilon^{\prime}\right)$ is uniquely determined.

Along the same monotone increasing curve $\varepsilon(\varepsilon)$, the $x$ support $\mathcal{X}_{f}$ in Def. 4 can be defined. Let $\boldsymbol{x}_{*} \triangleq \boldsymbol{x}^{(\infty)}(\mathbf{1}, 1)$ be the fixed point when $\varepsilon=1$. Then, a portion of the EBP EXIT curve can be constructed along a smooth and strictly increasing (w.r.t. the partial order) path, $\boldsymbol{x}(x) \in \mathcal{X}_{f}$, characterized by $x \in[0,1]$ with $\boldsymbol{x}(1)=\boldsymbol{x}_{*}$. Let $\varepsilon(\boldsymbol{x})$ be the $\varepsilon$-set defined in Def. 4. It has been shown in Lem. 3 that $\varepsilon(\boldsymbol{x})$ is unique. If $\varepsilon(\boldsymbol{x}(x))$ is smooth in $x$, then the EBP EXIT curve is given implicitly by $\left(\varepsilon(\boldsymbol{x}(x)), \mathrm{h}^{\mathrm{EBP}}(\boldsymbol{x}(x))\right)$, where $\mathrm{h}^{\mathrm{EBP}}(\boldsymbol{x}(x))$ is the associated EBP EXIT function [30], [26].

Definition 14: Given the EBP EXIT curve, the trial entropy $P(\boldsymbol{x})$ can be defined along the path $\boldsymbol{x}(x)$ with

$$
P(\boldsymbol{x}(x)) \triangleq P\left(\boldsymbol{x}_{*}\right)-\int_{x}^{1} \mathrm{~h}^{\mathrm{EBP}}(\boldsymbol{x}(t)) \varepsilon^{\prime}(\boldsymbol{x}(t)) \cdot \boldsymbol{x}^{\prime}(t) \mathrm{d} t .
$$

Note that the constant $P\left(\boldsymbol{x}_{*}\right)$ in (14) is determined by the system under consideration. For example, in the following applications, we know that $\boldsymbol{x}_{*}=\mathbf{1}$ and $P\left(\boldsymbol{x}_{*}\right)$ is equal to the design rate of the system [26].

Remark 7: The trial entropy is called path independent if $P\left(\boldsymbol{x}_{1}\left(x_{1}\right)\right)=P\left(\boldsymbol{x}_{2}\left(x_{2}\right)\right)$ for any two smooth paths $\boldsymbol{x}_{1}(x)$ and $\boldsymbol{x}_{2}(x)$ in $\mathcal{X}_{f}$ satisfying $\boldsymbol{x}_{1}\left(x_{1}\right)=\boldsymbol{x}_{2}\left(x_{2}\right)=\boldsymbol{x}$ for $x_{1}, x_{2} \in$ $[0,1]$. This is required for a well-defined trial entropy $P(\boldsymbol{x})$.

Definition 15 (c.f. [33, Conj. 1]): For a $P(\boldsymbol{x})$ that is path independent, the Maxwell Threshold $\varepsilon^{\mathrm{Max}}$ is defined by

$$
\varepsilon^{\operatorname{Max}}=\inf \left\{\varepsilon \in \mathcal{E} \mid \max _{\boldsymbol{x} \in \mathcal{F}(\varepsilon)} P(\boldsymbol{x})>0\right\} .
$$

Remark 8: In [33, Conj. 1], the Maxwell threshold is defined implicitly as the conjectured MAP threshold for irregular LDPC codes on the BEC. When the MAP threshold is determined by stability, the definition in [33, Conj. 1], however, does not identify the stability threshold correctly. This can be repaired simply by replacing the interval $(0,1]$ in their conjecture with the interval $[0,1]$ and defining the $\varepsilon$ associated with the fixed point $x=0$ to be the limit as $x \rightarrow 0$. For irregular LDPC codes, Def. 15 is equivalent to the corrected definition in [33, Conj. 1]. We also believe that it is the correct extension for our more general setup.

\section{B. Noisy Slepian-Wolf Problem with Erasures}

Two correlated discrete memoryless sources are encoded by two independent linear encoding functions, which are then transmitted through two independent erasure channels with erasure rates $\varepsilon_{1}$ and $\varepsilon_{2}$, respectively. We consider an erasure correlation model between the two sources. More specifically, let $Z$ be a Bernoulli- $p$ random variable such that the two sources are the same Bernoulli- $\frac{1}{2}$ random variable if $Z=1$ and are i.i.d. Bernoulli- $\frac{1}{2}$ random variables if $Z=0$. The decoder is assumed to have access to the side information $Z$.

Assume that the $i$-th source sequence is mapped into an LDPC code with degree distribution (d.d.) $\left(\lambda_{i}, \rho_{i}\right)$ and design rate $\gamma=1-L_{i}^{\prime}(1) / R_{i}^{\prime}(1)$ using a punctured systematic encoder of rate $\gamma /(1-\gamma)$. The fraction of punctured systematic bits is $\gamma$ (see [7] for details). Let $x_{1}^{(\ell)}$ (resp. $x_{2}^{(\ell)}$ ) be the average erasure rate of messages, from bit nodes to check nodes, corresponding to source 1 (resp. 2) and $\boldsymbol{x}^{(\ell)}=\left[x_{1}^{(\ell)}, x_{2}^{(\ell)}\right]$. Let $\mathcal{C}:[0,1] \rightarrow[0,1]^{2}, \varepsilon \mapsto\left[\varepsilon_{1}(\varepsilon), \varepsilon_{2}(\varepsilon)\right]$ with $\left[\varepsilon_{1}(0), \varepsilon_{2}(0)\right]=\mathbf{0}$, be continuous and monotonically increasing. The $\mathrm{DE}$ recursion in [7] is easily generalized to asymmetric d.d.s and can be written in the form of (1) with

$$
\begin{aligned}
\psi(x ; \varepsilon) & \triangleq(1-\gamma) \varepsilon+\gamma(1-p+p x), \\
\boldsymbol{f}(\boldsymbol{x} ; \varepsilon) & \triangleq\left[\psi\left(L_{2}\left(x_{2}\right) ; \varepsilon_{1}(\varepsilon)\right) \lambda_{1}\left(x_{1}\right), \psi\left(L_{1}\left(x_{1}\right) ; \varepsilon_{2}(\varepsilon)\right) \lambda_{2}\left(x_{2}\right)\right], \\
\boldsymbol{g}(\boldsymbol{x}) & \triangleq\left[1-\rho_{1}\left(1-x_{1}\right), 1-\rho_{2}\left(1-x_{2}\right)\right] .
\end{aligned}
$$

Using Def. 2 and $\boldsymbol{D}=\operatorname{diag}\left(L_{1}^{\prime}(1), L_{2}^{\prime}(1)\right)$, one finds that

$$
\begin{aligned}
F(\boldsymbol{x} ; \varepsilon)= & \psi\left(L_{1}\left(x_{1}\right) ; \varepsilon_{2}(\varepsilon)\right) L_{2}\left(x_{2}\right) \\
& +\psi\left(L_{2}\left(x_{2}\right) ; \varepsilon_{1}(\varepsilon)\right) L_{1}\left(x_{1}\right)-\gamma p L_{1}\left(x_{1}\right) L_{2}\left(x_{2}\right), \\
G(\boldsymbol{x})= & \sum_{k=1}^{2} L_{k}^{\prime}(1)\left(x_{k}+\frac{R_{k}\left(1-x_{k}\right)-1}{R_{k}^{\prime}(1)}\right) .
\end{aligned}
$$


For asymmetric d.d.s, one can also generalize both the trial entropy $P(\boldsymbol{x})$, from [26, Lem. 4], and the mapping $\varepsilon(\boldsymbol{x})=$ $\left[\varepsilon^{[1]}(\boldsymbol{x}), \varepsilon^{[2]}(\boldsymbol{x})\right]$, from [26, Sec. II-A]. This gives

$U(\boldsymbol{x} ; \varepsilon)=(1-\gamma)\left(\left(\varepsilon(\boldsymbol{x})-\left[\varepsilon_{1}(\varepsilon), \varepsilon_{2}(\varepsilon)\right]\right) \boldsymbol{L}(\boldsymbol{g}(\boldsymbol{x}))^{\boldsymbol{\top}}-P(\boldsymbol{x})\right)$,

where $\boldsymbol{L}(\boldsymbol{g}(\boldsymbol{x}))=\left[L_{1}\left(g_{1}\left(x_{1}\right)\right), L_{2}\left(g_{2}\left(x_{2}\right)\right)\right]$. Since $Q(\boldsymbol{x}) \triangleq$ $U(\boldsymbol{x} ; \varepsilon(\boldsymbol{x}))$, substituting $\varepsilon \mapsto \varepsilon(\boldsymbol{x})$ into $U(\boldsymbol{x} ; \varepsilon)$ shows that

$$
Q(\boldsymbol{x})=-(1-\gamma) P(\boldsymbol{x}) .
$$

Lemma 12: Let $\varepsilon^{*}$ be the potential threshold from (7). Then, $\varepsilon^{*}=\varepsilon^{\text {Max }}$, where $\varepsilon^{\text {Max }}$ is the Maxwell threshold defined by (12).

Proof: By substituting (13) into (12), it can be shown that

$$
\varepsilon^{\operatorname{Max}} \triangleq \inf \left\{\varepsilon \in \mathcal{E} \mid \min _{\boldsymbol{x} \in \mathcal{F}(\varepsilon)} Q(\boldsymbol{x})<0\right\} .
$$

Since the condition in (7) is the complement of the condition in (14) and the minimum $\min _{\boldsymbol{x} \in \mathcal{F}(\varepsilon)} Q(\boldsymbol{x})$ is strictly decreasing in $\varepsilon$, these thresholds are equal.

Corollary 2: Applying Theorem 1 shows that, if $\varepsilon<\varepsilon^{\text {Max }}$ and $w>K_{\boldsymbol{f}, \boldsymbol{g}} / \Delta E(\varepsilon)$, then the SC Slepian-Wolf DE recursion must converge to the zero matrix.

Remark 9: For special cases, one can use the methods in [26], [31], [32] to show that the Maxwell threshold defined above is an upper bound on the MAP threshold. These references also show that, for regular LDPC codes with fixed rate and increasing degrees, the upper bound approaches the information-theoretic limit. Therefore, SC regular LDPC codes are universal (e.g., see [7]) for this problem.

\section{Erasure Multiple-Access Channel}

We consider the two-user MAC channel with erasure noise (EMAC) from [25]. Let the inputs be $X^{[1]}, X^{[2]} \in\{ \pm 1\}$ and the output be

$$
Y=\left\{\begin{array}{ll}
X^{[1]}+X^{[2]} & \text { with probability } 1-\varepsilon, \\
? & \text { with probability } \varepsilon
\end{array} .\right.
$$

Assume that the source sequences are encoded by LDPC codes with d.d.s $\left(\lambda_{1}, \rho_{1}\right)$ and $\left(\lambda_{2}, \rho_{2}\right)$. Let $x_{1}^{(\ell)}$ (resp. $\left.x_{2}^{(\ell)}\right)$ be the average erasure rate of messages from bit nodes to check nodes corresponding to user 1 (resp. 2) and $\boldsymbol{x}^{(\ell)}=\left[x_{1}^{(\ell)}, x_{2}^{(\ell)}\right]$. In [26], the DE recursion is written as (1), with

$$
\begin{aligned}
\psi(x ; \varepsilon) & \triangleq \varepsilon+(1-\varepsilon) x / 2, \\
\boldsymbol{f}(\boldsymbol{x} ; \varepsilon) & \triangleq\left[\psi\left(L_{2}\left(x_{2}\right) ; \varepsilon\right) \lambda_{1}\left(x_{1}\right), \psi\left(L_{1}\left(x_{1}\right) ; \varepsilon\right) \lambda_{2}\left(x_{2}\right)\right], \\
\boldsymbol{g}(\boldsymbol{x}) & \triangleq\left[1-\rho_{1}\left(1-x_{1}\right), 1-\rho_{2}\left(1-x_{2}\right)\right] .
\end{aligned}
$$

Using Def. 2 and $\boldsymbol{D}=\operatorname{diag}\left(L_{1}^{\prime}(1), L_{2}^{\prime}(1)\right)$, one finds that

$$
\begin{aligned}
F(\boldsymbol{x} ; \varepsilon) & =\varepsilon\left[L_{1}\left(x_{1}\right)+L_{2}\left(x_{2}\right)\right]+(1-\varepsilon) L_{1}\left(x_{1}\right) L_{2}\left(x_{2}\right) / 2, \\
G(\boldsymbol{x}) & =\sum_{k=1}^{2} L_{k}^{\prime}(1)\left(x_{k}+\frac{R_{k}\left(1-x_{k}\right)-1}{R_{k}^{\prime}(1)}\right) .
\end{aligned}
$$

Let the trial entropy, $P(\boldsymbol{x})$, and the $\varepsilon(\boldsymbol{x})$ be defined by [26, Lem. 10]. Then, $U(\boldsymbol{x} ; \varepsilon)$ equals

$$
(\varepsilon(\boldsymbol{x})-\varepsilon)\left[\boldsymbol{L}(\boldsymbol{g}(x)) \mathbf{1}^{\top}-\frac{1}{2} L_{1}\left(g_{1}\left(x_{1}\right)\right) L_{2}\left(g_{2}\left(x_{2}\right)\right)\right]-P(\boldsymbol{x}),
$$

and substituting $\varepsilon \mapsto \varepsilon(\boldsymbol{x})$ implies that $Q(\boldsymbol{x})=-P(\boldsymbol{x})$. Similar to Lemma 12 , it can also be shown that $\varepsilon^{*}=\varepsilon^{\mathrm{Max}}$.

Corollary 3: Applying Theorem 1 shows that, if $\varepsilon<\varepsilon^{\mathrm{Max}}$ and $w>K_{\boldsymbol{f}, \boldsymbol{g}} / \Delta E(\varepsilon)$, then the $\mathrm{SC} \mathrm{DE}$ recursion for the erasure MAC channel must converge to the zero matrix.

Remark 10: For special cases, one can use the methods in [26], [31], [32] to show that the Maxwell threshold defined above is an upper bound on the MAP threshold. These references also show that, for regular LDPC codes with fixed rate and increasing degrees, the upper bound approaches the information-theoretic limit of the EMAC channel.

\section{General Protograph Codes on the BEC}

Consider the protograph ensemble [34] defined by an $m \times n$ protograph parity-check matrix $H$ (e.g., $H=\left[\begin{array}{ll}3 & 3\end{array}\right]$ defines a $(3,6)$-regular code) and let $[k]$ denote the set $\{1,2, \ldots, k\}$. Let the dimension of the recursion, $d$, equal the number of non-zero entries in $H$ and let the functions $r:[d] \rightarrow[m]$, $c:[d] \rightarrow[n]$, and $e:[d] \rightarrow\{1,2, \ldots\}$ map the index of each non-zero entry to its row, column, and value (i.e., $e(k)=$ $H_{r(k), c(k)}$ for $\left.k \in[d]\right)$. Let $\varepsilon_{j}(\varepsilon)$ be the erasure probability of the $j$-th bit node in the protograph as a function of the channel parameter $\varepsilon$. Then, the bit- and check-node DE update functions $\boldsymbol{f}(\boldsymbol{x}), \boldsymbol{g}(\boldsymbol{x})$ are given by

$$
\begin{aligned}
f_{k}(\boldsymbol{x} ; \varepsilon) & =\varepsilon_{c(k)}(\varepsilon) \prod_{i \in[d]: c(i)=c(k)} x_{i}^{e(i)-\delta_{i, k}} \\
g_{k}(\boldsymbol{x}) & =1-\prod_{j \in[d]: r(j)=r(k)}\left(1-x_{j}\right)^{e(j)-\delta_{i, k}},
\end{aligned}
$$

where $\delta_{i, j}$ is the Kronecker delta function. From this, we make an educated guess that the bit- and check-node potentials are

$$
\begin{aligned}
F(\boldsymbol{x} ; \varepsilon) & =\sum_{j=1}^{n} \varepsilon_{j}(\varepsilon) \prod_{i \in[d]: c(i)=j} x_{i}^{e(i)} \\
G(\boldsymbol{x}) & =\sum_{k=1}^{d} e(k) x_{k}-\sum_{i=1}^{m}\left[1-\prod_{j \in[d]: r(j)=i}\left(1-x_{j}\right)^{e(j)}\right] .
\end{aligned}
$$

Since each non-zero entry in $H$ appears in only one row and one column, it is easy to verify that

$$
\frac{\mathrm{d}}{\mathrm{d} x_{k}} F(\boldsymbol{x} ; \varepsilon)=e(k) f_{k}(\boldsymbol{x} ; \varepsilon) \text { and } \frac{\mathrm{d}}{\mathrm{d} x_{k}} G(\boldsymbol{x})=e(k) g_{k}(\boldsymbol{x}) \text {. }
$$

This shows that one can choose $\boldsymbol{D}=\operatorname{diag}(e(1), \ldots, e(d))$ and then apply Def. 2 to define a potential function for the protograph DE update. It is easy to verify that the DE equations comprise a vector admissible system. Therefore, we conjecture that the fixed-point potential, $Q(\boldsymbol{x})$, will also be a scalar multiple of the trial entropy defined by integration of the BP EXIT curve [30].

\section{CONCLusions}

Based on the work in [22], a new theorem is presented that provides a simple proof of threshold saturation for a broad class of vector recursions. The conditions of the theorem are verified for the density-evolution equations associated with: (i) irregular LDPC codes for a Slepian-Wolf problem with erasures, (ii) irregular LDPC codes on the erasure multiple-access 
channel, and (iii) protograph codes on BEC. This provides the first proof of threshold saturation for these systems. Along with the results in [26], [31], [32], this also shows that SC codes are universal (e.g., see [7]) for the noisy Slepian-Wolf problem with erasures.

Acknowledgment: The authors thank Kenta Kasai for identifying a few subtle errors in an earlier version of this manuscript and Andrew J. Young for suggestions on and assistance with the final version.

\section{REFERENCES}

[1] J. Felstrom and K. S. Zigangirov, "Time-varying periodic convolutional codes with low-density parity-check matrix," IEEE Trans. Inform. Theory, vol. 45 , no. 6, pp. 2181-2191, 1999.

[2] A. Sridharan, M. Lentmaier, D. J. Costello, and K. S. Zigangirov, "Convergence analysis of a class of LDPC convolutional codes for the erasure channel," in Proc. Annual Allerton Conf. on Commun., Control, and Comp., (Monticello, IL), pp. 953-962, 2004.

[3] M. Lentmaier, A. Sridharan, K. S. Zigangirov, and D. J. Costello, "Terminated LDPC convolutional codes with thresholds close to capacity," in Proc. IEEE Int. Symp. Inform. Theory, (Adelaide, Australia), pp. 13721376, 2005 .

[4] M. Lentmaier, A. Sridharan, D. J. Costello, and K. S. Zigangirov, "Iterative decoding threshold analysis for LDPC convolutional codes," IEEE Trans. Inform. Theory, vol. 56, pp. 5274-5289, Oct. 2010.

[5] S. Kudekar, C. Méasson, T. Richardson, and R. Urbanke, "Threshold saturation on BMS channels via spatial coupling," in Proc. Int. Symp. on Turbo Codes \& Iterative Inform. Proc., pp. 309-313, Sept. 2010.

[6] V. Rathi, R. Urbanke, M. Andersson, and M. Skoglund, "Rateequivocation optimally spatially coupled LDPC codes for the BEC wiretap channel," in Proc. IEEE Int. Symp. Inform. Theory, (St. Petersburg, Russia), pp. 2393-2397, July 2011.

[7] A. Yedla, H. D. Pfister, and K. R. Narayanan, "Universality for the noisy Slepian-Wolf problem via spatial coupling," in Proc. IEEE Int. Symp. Inform. Theory, (St. Petersburg, Russia), pp. 2567-2571, July 2011.

[8] S. Kudekar and K. Kasai, "Threshold saturation on channels with memory via spatial coupling," in Proc. IEEE Int. Symp. Inform. Theory, (St. Petersburg, Russia), pp. 2562-2566, July 2011.

[9] P. S. Nguyen, A. Yedla, H. D. Pfister, and K. R. Narayanan, "Spatiallycoupled codes and threshold saturation on intersymbol-interference channels." to be submitted to IEEE Trans. on Inform. Theory, [Online]. Available: http://arxiv.org/abs/1107.3253, 2012.

[10] P. S. Nguyen, A. Yedla, H. D. Pfister, and K. R. Narayanan, "Threshold saturation of spatially-coupled codes on intersymbol-interference channels," in Proc. IEEE Int. Conf. Commun., (Ottawa, Canada), pp. 22092214, June 2012.

[11] S. Kudekar, T. Richardson, and R. Urbanke, "Spatially coupled ensembles universally achieve capacity under belief propagation." Arxiv preprint arXiv:1201.2999, 2012.

[12] S. Kudekar, T. J. Richardson, and R. L. Urbanke, "Threshold saturation via spatial coupling: Why convolutional LDPC ensembles perform so well over the BEC," IEEE Trans. Inform. Theory, vol. 57, no. 2, pp. 803834, 2011.

[13] M. Lentmaier and G. P. Fettweis, "On the thresholds of generalized LDPC convolutional codes based on protographs," in Proc. IEEE Int. Symp. Inform. Theory, (Austin, TX), pp. 709-713, 2010.

[14] S. H. Hassani, N. Macris, and R. Urbanke, "Coupled graphical models and their thresholds," in Proc. IEEE Inform. Theory Workshop, (Dublin, Ireland), pp. 1-5, 2010.
[15] S. H. Hassani, N. Macris, and R. Urbanke, "Chains of mean-field models," J. Stat. Mech., p. P02011, 2012.

[16] K. Takeuchi, T. Tanaka, and T. Kawabata, "Improvement of BP-based CDMA multiuser detection by spatial coupling," in Proc. IEEE Int. Symp. Inform. Theory, (St. Petersburg, Russia), pp. 1489-1493, July 2011.

[17] C. Schlegel and D. Truhachev, "Multiple access demodulation in the lifted signal graph with spatial coupling," in Proc. IEEE Int. Symp. Inform. Theory, (St. Petersburg, Russia), pp. 2989-2993, July 2011.

[18] Y.-Y. Jian, H. D. Pfister, and K. R. Narayanan, "Approaching capacity at high rates with iterative hard-decision decoding," in Proc. IEEE Int. Symp. Inform. Theory, pp. 2696-2700, 2012.

[19] S. Kudekar and H. D. Pfister, "The effect of spatial coupling on compressive sensing," in Proc. Annual Allerton Conf. on Commun., Control, and Comp., (Monticello, IL), pp. 347-353, Oct. 2010.

[20] F. Krzakala, M. Mézard, F. Sausset, Y. Sun, and L. Zdeborová, "Statistical physics-based reconstruction in compressed sensing." Arxiv preprint arXiv:1109.4424, Sept. 2011.

[21] D. L. Donoho, A. Javanmard, and A. Montanari, "Informationtheoretically optimal compressed sensing via spatial coupling and approximate message passing." Arxiv preprint arXiv:1112.0708, Dec. 2011.

[22] A. Yedla, Y.-Y. Jian, P. S. Nguyen, and H. D. Pfister, "A simple proof of threshold saturation for coupled scalar recursions," in Proc. Int. Symp. on Turbo Codes \& Iterative Inform. Proc., 2012. Arxiv preprint arXiv:1204.5703, 2012.

[23] K. Takeuchi, T. Tanaka, and T. Kawabata, "A phenomenological study on threshold improvement via spatial coupling." Arxiv preprint arXiv:1102.3056, 2011.

[24] S. Kudekar, T. Richardson, and R. Urbanke, "Wave-like solutions of general one-dimensional spatially coupled systems." submitted to IEEE Trans. on Inform. Theory, [Online]. Available: http://arxiv.org/abs/1208.5273, Aug. 2012.

[25] S. Kudekar and K. Kasai, "Spatially coupled codes over the multiple access channel," in Proc. IEEE Int. Symp. Inform. Theory, (St. Petersburg, Russia), pp. 2816-2820, July 2011.

[26] P. S. Nguyen, A. Yedla, H. D. Pfister, and K. R. Narayanan, "On the maximum a posteriori decoding thresholds of multiuser systems with erasures," in Proc. IEEE Int. Symp. Inform. Theory, (Cambridge, MA), pp. 2711-2715, July 2012.

[27] J. R. Magnus and H. Neudecker, Matrix Differential Calculus With Applications in Statistics and Econometrics. Wiley Series in Probability and Statistics, John Wiley, 1999.

[28] P. Vontobel, "Message-passing iterative decoding and linear programming decoding: news and views." Lectures at 2009 ACoRN Spring School, University of South Australia, Adelaide, Australia. [Available online at www.pseudocodewords.info], Nov. 2009.

[29] J. M. Walsh and P. A. Regalia, "On the relationship between belief propagation decoding and joint maximum likelihood detection," IEEE Trans. Commun., vol. 58, no. 10, pp. 2753-2758, 2010.

[30] T. J. Richardson and R. L. Urbanke, Modern Coding Theory. Cambridge University Press, 2008.

[31] P. S. Nguyen, Advanced Coding Techniques with Applications to Storage Systems. PhD thesis, Texas A\&M University, College Station, TX, 2012.

[32] A. Yedla, Universality for Multi-Terminal Problems via Spatial Coupling. PhD thesis, Texas A\&M University, College Station, TX, 2012.

[33] C. Méasson, A. Montanari, and R. L. Urbanke, "Maxwell construction: The hidden bridge between iterative and maximum a posteriori decoding," IEEE Trans. Inform. Theory, vol. 54, pp. 5277-5307, Dec. 2008.

[34] J. Thorpe, "Low-density parity-check (LDPC) codes constructed from protographs," IPN Progress Report, vol. 42, Aug. 2003. 